CONCISE REPORT

\title{
Lupus thrombocytopenia: clinical implications and prognostic significance
}

\section{P D Ziakas, S Giannouli, E Zintzaras, A G Tzioufas, M Voulgarelis}

Objectives: To clarify clinical manifestations, association with disease activity, and prognostic impact of thrombocytopenia using simple and reliable indices.

Methods: 632 patients were reviewed retrospectively. Fifty patients with thrombocytopenia were included as cases and matched with 100 control patients. Clinical manifestations at first thrombocytopenic episode were recorded. Classification criteria at diagnosis, basic immunological profiles, disease activity (ECLAM), and end organ damage (SLICC) were recorded.

Results: 29/50 (58\%) had thrombocytopenia at diagnosis of lupus. Haemorrhagic manifestations were associated with the degree of thrombocytopenia $(p<0.001)$. Anticardiolipin antibodies were not related to the degree of thrombocytopenia or the severity of haemorrhagic manifestations. Megakaryocytes were normal or increased in 26/28 (93\%) bone marrow specimens, indicating peripheral platelet destruction. Patients with high disease activity were more thrombocytopenic than controls $(O R=2.61,95 \% \mathrm{Cl} 1.13$ to 5.96, $\mathrm{p}=0.009$ ). Patients with low $\mathrm{C} 3$ or $\mathrm{CH}_{50}$ were more likely to be thrombocytopenic $(O R=2.36,95 \% \mathrm{Cl} 1.05$ to 5.26, $p=0.029$ ). Median SLICC for lupus patients with thrombocytopenia was 2 (range 0-11) compared with 1 (range $0-12$ ) for controls $(p<0.001)$. No deaths occurred during thrombocytopenic episodes.

Conclusions: Thrombocytopenia is not directly associated with end organ damage and mortality, but defines a subgroup of patients with higher morbidity and is thus a major complication of systemic lupus erythematosus, affecting overall prognosis.

$\mathrm{T}$ hrombocytopenia due to peripheral consumption of platelets is a common clinical manifestation in systemic lupus erythematosus (SLE), ranging from $7 \%$ to $30 \%$ in large reported series. Apart from diverse aetiologies in lupus thrombocytopenia, its clinical implications and prognostic impact still remain a matter of debate. Results of studies on the prognostic significance of thrombocytopenia in patients with SLE have been contradictory. ${ }^{1-3}$ In an endeavour to explain these disparate observations, a retrospective study was undertaken to analyse basic clinical and immunological characteristics, end organ damage, and disease activity in thrombocytopenic patients with SLE.

\section{PATIENTS AND METHODS}

All available 632 medical records of patients with SLE were reviewed to confirm the patients' eligibility and gather demographic and clinical data from the time of diagnosis to enrolment. Case documentation, assessment of disease activity, damage events, and therapeutic interventions were made by the same group of rheumatologists throughout. Patients with SLE who had at least one episode of thrombocytopenia, defined as a platelet count of $<100 \times 10^{9} / 1$, were included in the study as cases, according to standard criteria. ${ }^{4}$ Each case was matched for age, sex, and disease duration with two patients with SLE who never developed thrombocytopenia.

\section{Variables}

Age, sex, clinical presentation, disease duration, disease activity, autoantibody profile, and organ damage were recorded. The criteria of the American College of Rheumatology for classification ${ }^{4}$ were obtained from all patients at the time of diagnosis. Disease activity was measured with the European Consensus Lupus Activity Measurement (ECLAM) score $^{6}$ calculated at the onset of thrombocytopenia in the case group. In this group, thrombocytopenia was not included in the ECLAM score in order to obtain a more accurate comparison of SLE activity between the two groups. In the control group the ECLAM score was obtained at the same interval from diagnosis ( \pm 1 month).

Organ damage was recorded at the end of follow up according to the Systemic Lupus International Collaborating Clinics (SLICC) Damage Index ${ }^{7}$ and was further classified into five broad categories as renal, neuropsychiatric, cardiovascular, pulmonary, and treatment related. The deceased cumulative damage was scored just before their death.

Antinuclear antibodies were determined by indirect immunofluorescence; anti-dsDNA by a standard enzyme linked immunosorbent assay (ELISA); anti-extractable nuclear antigens antibodies by counterimmunoelectrophoresis; serum C3 by nephelometry; $\mathrm{CH}_{50}$ by Autokit $\mathrm{CH}_{50}$, a commercial liposome immunoassay; and anticardiolipin antibodies (ACA) by a standard ELISA. Anti-dsDNA and serum C3 were measured frequently. ACA were tested at periods of disease activity. Complement levels and anti-dsDNA activity at thrombocytopenia in the case group and at matched disease duration for the control group were obtained for analysis.

Platelet number at the onset of thrombocytopenia, haemorrhagic manifestations, and number of relapses were also recorded for the case group. The presence of megakaryocytes was assessed by reviewing available bone marrow biopsy specimens at the onset of thrombocytopenia.

Various treatments were also recorded in both groups and broadly classified into low and high intensity regimens: the former comprised low dose corticosteroids with or without methotrexate or azathioprine and the latter, high dose corticosteroids with or without intravenous cyclophosphamide.

\section{Statistical analysis}

Continuous variables were compared using the MannWhitney U test. Categorical variables were compared using

Abbreviations: $\mathrm{ACA}$, anticardiolipin antibodies; $\mathrm{Cl}$, confidence interval; ECLAM, European Consensus Lupus Activity Measurement; ELISA, enzyme linked immunosorbent assay; OR, odds ratio; SLE, systemic lupus erythematosus; SLICC, Systemic Lupus International Collaborating Clinics 
Table 1 Demographic, clinical, and autoimmune profile among thrombocytopenic group and control group

\begin{tabular}{|c|c|c|c|c|}
\hline & Cases & Controls & Odds ratio & $95 \% \mathrm{Cl}$ \\
\hline \multicolumn{5}{|l|}{ Demographic data } \\
\hline $\operatorname{Sex}(F / M)$ & $47 / 3$ & $94 / 6$ & & \\
\hline Age (years), mean (SD) & $27.4(13.3)$ & $28.2(12.5)$ & & \\
\hline Time from diagnosis (years), mean (SD) & $11.7(5.9)$ & $11.6(5.6)$ & & \\
\hline \multicolumn{5}{|l|}{ Clinical data } \\
\hline ECLAM score median (min-max) & $3(1-8)$ & $3(0-7)$ & & \\
\hline ECLAM $\geqslant 4^{*}$ & $19 / 50(38)$ & $19 / 100(19)$ & 2.61 & 1.13 to 5.96 \\
\hline Malar rash & $23 / 50(46)$ & $39 / 100$ (39) & 1.33 & 0.63 to 2.79 \\
\hline Discoid rash & $2 / 50(4)$ & $1 / 100(1)$ & 4.12 & 0.20 to 245.73 \\
\hline Photosensitivity & $22 / 50$ (44) & $37 / 100$ (37) & 1.33 & 0.63 to 2.81 \\
\hline Oral ulcers & $10 / 50(20)$ & $28 / 100(28)$ & 0.64 & 0.25 to 1.54 \\
\hline Arthritis & $26 / 50(52)$ & $60 / 100(60)$ & 0.72 & 0.34 to 1.51 \\
\hline Serositis & $7 / 50(14)$ & $26 / 100(26)$ & 0.46 & 0.15 to 1.22 \\
\hline Renal disease§ & $10 / 50(20)$ & $26 / 100(26)$ & 0.71 & 0.27 to 1.71 \\
\hline Neurological disorder & $8 / 50(16)$ & $17 / 100(17)$ & 0.92 & 0.32 to 2.50 \\
\hline Haemolytic anaemia** & $9 / 50(18)$ & $17 / 100(17)$ & 1.07 & 0.38 to 2.80 \\
\hline Leucopenia $\left(<4 \times 10^{9} /\right.$ /) & $26 / 50(52)$ & $45 / 100(45)$ & 1.32 & 0.53 to 2.76 \\
\hline \multicolumn{5}{|l|}{ Antibody profile } \\
\hline ANA & $50 / 50(100)$ & $100 / 100(100)$ & & \\
\hline Anti-dsDNA & $28 / 50(56)$ & $54 / 100(54)$ & 1.08 & 0.51 to 2.2 \\
\hline Anti-Sm & $5 / 50(10)$ & $6 / 100(6)$ & 1.74 & 0.39 to 7.22 \\
\hline Anti-Ro(SSA) & $18 / 50(36)$ & $31 / 100(31)$ & 1.25 & 0.56 to 2.70 \\
\hline Anti-La(SSB) & $6 / 50(12)$ & $4 / 100(4)$ & 3.27 & 0.72 to 16.44 \\
\hline Anti-RNP & $10 / 50(20)$ & $11 / 100(11)$ & 2.02 & 0.70 to 5.70 \\
\hline Anticardiolipin & $14 / 50$ (28) & $20 / 100(20)$ & 1.55 & 0.64 to 3.65 \\
\hline $\lg G(>100 \mathrm{U})$ & $11 / 50$ (22) & $16 / 100(16)$ & & \\
\hline $\lg M(>100 \mathrm{U})$ & $10 / 50(20)$ & $11 / 100(11)$ & & \\
\hline Both & $7 / 50$ (14) & $9 / 100(9)$ & & \\
\hline Complement activity (low $\mathrm{C} 3$ or $\mathrm{CH}_{50}$ ) & $20 / 50(40)$ & $22 / 100(22)$ & 2.36 & 1.05 to 5.26 \\
\hline \multicolumn{5}{|l|}{ Treatmentt } \\
\hline Low intensity & $26 / 48(54)$ & $74 / 98(76)$ & & \\
\hline High intensity & $22 / 48(46)$ & $24 / 98(24)$ & & \\
\hline $\begin{array}{l}\text { Results are shown as No (\%) unless state } \\
{ }^{*} \mathrm{p}=0.009 \text {, estimated power } 75 \% ; \mathrm{tp}=0 \\
\text { (red blood cell, haemoglobin, granular, } \\
\text { metabolic rearrangements; **haemolytic }\end{array}$ & lar, mixed); & ald es, pycto & ... & $\begin{array}{l}\text { day or cellular co } \\
\text { of offending drug }\end{array}$ \\
\hline
\end{tabular}

Pearson's $\chi^{2}$ test, and odds ratios (ORs) with their corresponding $95 \%$ confidence intervals (CIs) were calculated. A multivariate logistic regression was used for adjusting parameter effects. The probabilities of damage-free and relapse-free intervals for different groups were determined by a Kaplan-Meier survival analysis, and curves were compared using the log rank test. Significance was defined as a $\mathrm{p}$ value $<0.05$. Data were analysed using the SPSS and Statistica software packages.

\section{RESULTS}

\section{Case-control analysis}

Fifty three $(8.4 \%)$ patients with SLE and thrombocytopenia were found. Two patients with thrombocytopenia due to documented infection and one with methotrexate related bone marrow toxicity were excluded from the analysis. Fifty patients with SLE with thrombocytopenia and 100 without were finally included. Twenty eight had acute thrombocytopenia with platelets falling sharply to very low levels. Twenty two had had chronic, mild thrombocytopenia for months or years. Eight had thrombocytopenia preceding the diagnosis of SLE. Table 1 summarises individual demographic, clinical, and antibody profiles.

The two groups did not differ significantly in their median ECLAM score $(p=0.12)$, but patients with high disease activity as denoted by an ECLAM $\geqslant 4$, were more likely to be thrombocytopenic than controls (OR $=2.61,95 \%$ CI 1.13 to 5.96, $\mathrm{p}=0.009)$. An analysis of complement activity showed similar results, and patients with low $\mathrm{C} 3$ or $\mathrm{CH}_{50}$ were more likely to be thrombocytopenic ( $\mathrm{OR}=2.36,95 \%$ CI 1.05 to $5.26, p=0.029$ ), indicating a more active disease in thrombocytopenic patients. In logistic regression, the odds ratio of

Table 2 Severity of haemorrhagic manifestations in patients with thrombocytopenia

\begin{tabular}{cccccc}
\hline & Grade 0 & Grade I & Grade II & Grade III & $\begin{array}{l}\text { Total } \\
\text { No (\%) }\end{array}$ \\
\hline Platelet level $\left(/ \mathrm{mm}^{3}\right)$ & & & & & \\
$50-100000$ & 22 & 1 & 4 & 0 & $27(54)$ \\
$20-50000$ & 6 & 2 & 1 & 0 & $9(18)$ \\
$<20000$ & 1 & 7 & 5 & 1 & $14(28)$ \\
Total, No (\%) & $29(58)$ & $10(20)$ & $10(20)$ & $1(2)$ & $50(100)$ \\
Anticardiolipin & 7 & 5 & 2 & 0 & $14(28)$ \\
\hline
\end{tabular}

Grade 0 =none; grade I= mucosal bleeding; grade $\|$ = gastrointestinal bleeding, genitourinary tract bleeding; grade $\mathrm{II}=$ central nervous system bleeding, pulmonary haemorrhage. 


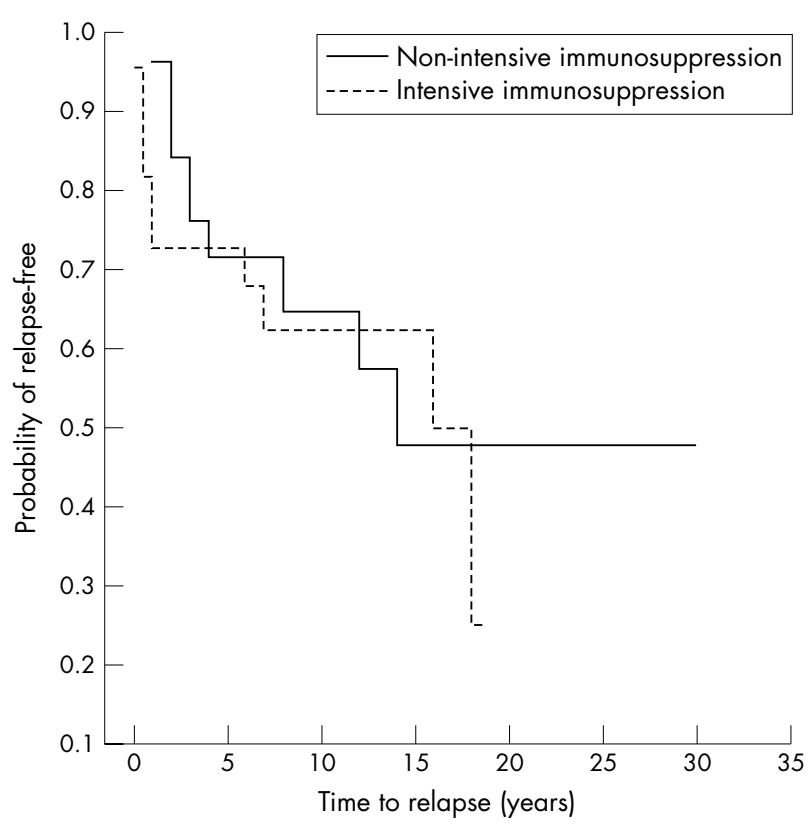

Figure 1 Kaplan-Meier curves for patients with SLE and thrombocytopenia and with and without intensive immunosuppression $(p=0.61)$, showing the probability of a relapse-free interval.

ECLAM $\geqslant 4$, adjusted for low complement, remained significant $(\mathrm{OR}=2.46(95 \% \mathrm{CI} 1.05$ to $5.75, \mathrm{p}=0.03))$. No other differences in the basic clinical and autoimmune profiles were seen between the two groups. Thrombocytopenia itself, appears as a cause of high intensity immunosuppressive treatment in comparison with the control group $(p=0.009$, table 1).

\section{Haemorrhagic manifestations}

Twenty nine $(58 \%)$ of 50 patients had thrombocytopenia at diagnosis of lupus. These patients did not differ significantly in their clinical and serological characteristics from those developing thrombocytopenia thereafter. At presentation of thrombocytopenia, there was a strong association between the severity of haemorrhagic manifestations and the degree of thrombocytopenia $(\mathrm{p}<0.001)$ (table 2$)$. The presence and isotype of ACA was unrelated to the severity of bleeding complications or the degree of thrombocytopenia.

\section{Bone marrow findings}

Twenty eight available bone marrow biopsy specimens during thrombocytopenic episodes were reviewed. Megakaryocytes were normal or increased in 26/28 (93\%) specimens indicating peripheral platelet destruction. Two (7\%) of 28 cases had a hypocellular and aplastic bone marrow, the former attributed to cytotoxic treatment and the latter to active disease.

\section{Treatment and relapse of thrombocytopenia}

Patients received high doses of corticosteroids intravenously or by mouth plus either pulse cyclophosphamide (17/53) or azathioprine (5/53) with or without intravenous globulin (12/ 53). Two of 53 patients were splenectomised. All patients finally achieved normal platelet counts.

Twenty two $(44 \%)$ of 50 patients had at least one relapse during the course of their disease, defined as a reduction in platelet count below the threshold of $100 \times 10^{9} / 1$ after achievement of a normal platelet count on at least two consecutive measurements. The probability of relapse-free intervals among thrombocytopenic subjects did not differ
Table 3 Cumulative organ damage at the end of follow up

\begin{tabular}{|c|c|c|}
\hline Damage & Controls & Cases \\
\hline 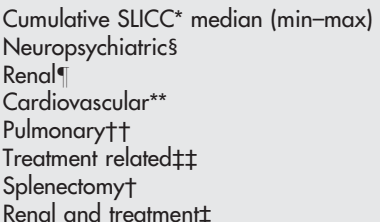 & $\begin{array}{l}1(0-12) \\
27 / 100(27) \\
7 / 100(7) \\
18 / 100(18) \\
5 / 100(5) \\
18 / 100(18) \\
0 / 100(0) \\
0 / 100(0)\end{array}$ & $\begin{array}{l}2(0-11) \\
13 / 50(26) \\
8 / 50(16) \\
16 / 50(32) \\
4 / 50(8) \\
14 / 50(28) \\
2 / 50(4) \\
6 / 50(12)\end{array}$ \\
\hline
\end{tabular}

Results are shown as No (\%).

${ }^{*} \mathrm{p}<0.001 ; \uparrow \mathrm{p}=0.044 ; \neq \mathrm{p}<0.001$, renal and treatment means damage from both in the same patient; §cognitive impairment, seizures, cerebrovascular accidents, psychosis, cranial or peripheral neuropathies; - $>3.5 \mathrm{~g}$ of protein/day or measured glomerular filtration rate $<50 \%$ or end stage renal disease (regardless of dialysis or transplantation; ${ }^{* *}$ coronary artery disease, myocardial infarctions, peripheral thromboses, pericarditis; †tpulmonary hypertension, pulmonary emboli; ¥¥cataract, diabetes mellitus, muscle atrophy, amenorrhoea.

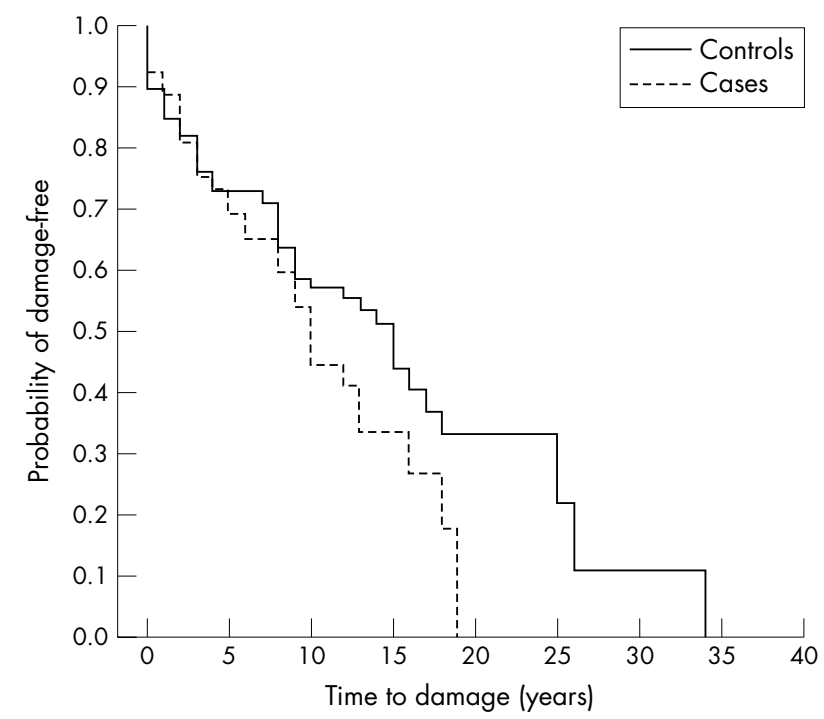

Figure 2 Kaplan-Meier curves for patients with SLE with thrombocytopenia (cases) and without thrombocytopenia (controls) $(p=0.14)$, showing the probability of a damage-free interval (time to first event). Median damage-free time for cases and controls were 10 and 14.5 years, respectively.

$(\mathrm{p}=0.61)$ with treatment: low $(\mathrm{n}=26)$ versus high intensity $(\mathrm{n}=22)$ (fig 1$)$.

ACA, severity of haemorrhagic manifestations, and high versus low ECLAM scores had no significant effect on the time to relapse (data not shown).

\section{Overall morbidity}

The two groups differed significantly in the number of end organ damage events, measured by the SLICC score at the end of follow up. Patients with thrombocytopenia had a median SLICC of $2(0-11)$ in contrast with controls who had a median of 1 (0-12), reflecting a more aggressive course among thrombocytopenic subjects $(\mathrm{p}<0.001)$. The two groups did not differ significantly in their organ involvement and treatment related sequelae. A striking exception was the coexistence of renal disease and treatment related complications among thrombocytopenic subjects in contrast with controls $(\mathrm{p}<0.001)$ (table 3$)$.

There was no difference in the probability of a damage-free interval between cases and controls (fig 2). Separate analysis 
for each category of damage was also performed. Results were not significant (data not shown).

\section{Deaths}

Eight patients died during follow up, four in the thrombocytopenic group. No deaths were seen during thrombocytopenic episodes or associated with haemorrhagic complications. Pulmonary emboli, pulmonary infection, and central nervous system involvement (stroke, status epilepticus) were the causes of death (data not shown).

\section{DISCUSSION}

Miller et al suggested that thrombocytopenia did not determine patients' subsequent course, but the coexistence of associated features such as glomerulonephritis and central nervous system involvement was a factor. ${ }^{8}$ Urowitz and Alger et al found no correlation with severe disease. ${ }^{9}{ }^{10}$ In contrast, other authors identified thrombocytopenia as an important prognostic feature. ${ }^{11-13}$ Ward et al added thrombocytopenia to nephritis and seizures as features which adversely affect survival. ${ }^{1}$ Recently, Sultan et al associated thrombocytopenic manifestations with active disease in other organs, though none of these patients succumbed as a direct result of this complication. ${ }^{14}$

Differences of outcome in the abovementioned studies may be attributed, at least in part, to different treatment approaches, heterogeneity of the population such as ethnicity, and study design. As far as we know, this is the first matched case-control study providing a more accurate estimation of clinical characteristics, autoimmune profile, and prognosis in thrombocytopenic patients with lupus. At the onset of thrombocytopenia, patients have higher disease activity than their matched controls as measured using the ECLAM score. A score of four or higher is associated with a higher incidence of thrombocytopenia. If complement is used as a single estimate of disease activity, low values are also associated with thrombocytopenia.

In most patients thrombocytopenia follows a benign course and only a few patients had severe bleeding. Splenectomy was only performed on $2 / 14$ (14\%) occasions of severe $\left(<20 \times 10^{9} / 1\right)$ thrombocytopenia, indicating a limited role for splenectomy. Although thrombocytopenia itself does not influence overall morbidity, cumulative organ damage, measured by the SLICC index, differs in the two groups. Patients with thrombocytopenia have a worse prognosis overall, as the SLICC index is a strong predictor of outcome and survival. ${ }^{15}$ Sites of organ involvement and treatment related complications do not differ between the groups. However, the coexistence of renal disease and treatment related complications is highly significant for the case group.

Our study has the limitations of a retrospective casecontrol study. However, all patients were attended by the same team of physicians at frequent intervals, medical records were routinely updated in detail, and possible sources of bias were limited. All patients were Greek in origin, so a separate analysis was unnecessary. The aforementioned conclusions, however, may not apply to other ethnic groups.

Our analysis indicates that lupus thrombocytopenia is a characteristic of active lupus. Although time to the first damage event does not differ between the two groups, thrombocytopenia emerges as a major indicator of cumulative morbidity among lupus patients. Thrombocytopenia should be considered as a reliable prognostic marker, identifying a subset of patients whose disease course is aggravated by end organ damage.

\section{ACKNOWLEDGEMENTS}

We are indebted to Professor HM Moutsopoulos, head of the department, for his inspiring recommendations and continuous support.

The study was supported by research funding from Genesis Pharma SA.

\section{Authors' affiliations}

P D Ziakas, S Giannouli, A G Tzioufas, M Voulgarelis, Department of Pathophysiology, University of Athens-Medical School, Athens, Greece E Zintzaras, Department of Biomathematics, University of Thessaly School of Medicine, Larissa, Greece

Correspondence to: Dr M Voulgarelis, University of Athens Medical School, Department of Pathophysiology, 75 M Asias Street, 11527, Athens, Greece; mvoulgar@med.voa.gr

Accepted 7 February 2005

\section{REFERENCES}

1 Ward MM, Pyun E, Studenski S. Mortality risks associated with specific clinical manifestations of systemic lupus erythematosus. Arch Intern Med 1996:56:1337-44.

2 Cervera R, Khamashta MA, Font J, Sebastiani GD, Gil A, Lavilla P, et al. Morbidity and mortality in systemic lupus erythematosus during a 5 -year period.A multicenter prospective study of 1,000 patients. European Working Party on Systemic Lupus Erythematosus. Medicine (Baltimore) 1999;78:167-75.

3 Mok CC, Lee KW, Ho CT, Lau CS, Wong RW. A prospective study of survival and prognostic indicators of systemic lupus erythematosus in a southern Chinese population. Rheumatology (Oxford) 2000;39:399-406.

4 Tan EM, Cohen AS, Fries JF, Masi AT, McShane DJ, Rothfield NF, et al. The 1982 revised criteria for the classification of systemic lupus erythematosus. Arthritis Rheum 1982;25:1271-7.

5 Frederiksen $\mathbf{H}$, Schmidt $\mathrm{K}$. The incidence of idiopathic thrombocytopenic purpura in adults increases with age. Blood 1999;94:909-13.

6 Mosca M, Bencivelli W, Vitali C, Carrai P, Neri R, Bombardieri S. The validity of the ECLAM index for the retrospective evaluation of disease activity in systemic lupus erythematosus. Lupus 2000;9:445-50.

7 Thumboo J, Lee HY, Fong KY, Chan SP, Chapman CA, Leong KH, et al. Accuracy of medical record scoring of the SLICC/ACR damage index for systemic lupus erythematosus. Lupus 2000;9:358-62.

8 Miller MH, Urowitz MB, Gladman DD. The significance of thrombocytopenia in systemic lupus erythematosus. Arthritis Rheum 1983;26:1181-6.

9 Urowitz MB. SLE subsets - divide and conquer. J Rheumatol 1977;4:332-3.

10 Alger M, Alarcon-Segovia D, Rivero SJ. Hemolytic anemia and thrombocytopenic purpura : two related subsets of systemic lupus erythematosus. J Rheumatol 1977;4:351-7.

11 Pistiner M, Wallace DJ, Nessim S, Metzger AL, Klinenberg JR. Lupus erythematosus in the 1980s: a survey of 570 patients. Semin Arthritis Rheum $1991 ; 21: 55-64$.

12 Swaak AJ, Nossent JC, Bronsveld W, Van Rooyen A, Nieuwenhuys EJ Theuns $L$, et al. Systemic lupus erythematosus. I. Outcome and survival: Dutch experience with 110 patients studied prospectively, Ann Rheum Dis 1989;48:447-54.

13 Reveille JD, Bartolucci A, Alarcon GS. Prognosis in systemic lupus erythematosus. Negative impact of increasing age at onset, black race, and thrombocytopenia, as well as causes of death. Arthritis Rheum 1990;33:37-48.

14 Sultan SM, Begum S, Isenberg DA. Prevalence, patterns of disease and outcome in patients with systemic lupus erythematosus who develop severe haematological problems. Rheumatology (Oxford) 2003;42:230-4.

15 Nived O, Jonsen A, Bengtsson AA, Bengtsson C, Sturfelt G. High predictive value of the Systemic Lupus International Collaborating Clinics/American College of Rheumatology damage index for survival in systemic lupus erythematosus. J Rheumatol 2002;29:1398-400. 